\title{
Some global results for the Degn-Harrison system with diffusion
}

\author{
Benedetta Lisena
}

Dipartimento di Matematica, Universitá degli studi di Bari, 70125 Bari, ITALY

\begin{abstract}
.
This paper considers the Degn-Harrison reaction-diffusion system subject to homogeneous Neumann boundary conditions in a smooth and bounded domain. Using the presence of contracting rectangles and the method of Lyapunov, we establish sufficient conditions for the global asymptotic stability of the unique constant steady state.
\end{abstract}

Mathematics Subject Classification (2010). 35K52, 35E15, 35Q92.

Keywords: Reaction-diffusion; Asymptotic stability; Invariant rectangle; Degn-Harrison model.

\section{Introduction}

Various biological and chemical models consist of systems of semilinear diffusion equations. They are introduced to explain many realistic phenomena. In this paper we consider the reaction-diffusion system

$$
\left\{\begin{array}{l}
\frac{\partial u}{\partial t}=d_{1} \Delta u+a-u-\frac{u v}{1+k u^{2}} \quad x \in \Omega, \quad t>0 \\
\frac{\partial v}{\partial t}=d_{2} \Delta v+b-\frac{u v}{1+k u^{2}}
\end{array}\right.
$$

where $\Omega$ is a bounded domain in $\mathbf{R}^{n}$ with smooth boundary. The corresponding ODE model was proposed by Degn and Harrison[4] to describe the respiratory behavior of a Klebsiella Aerogenes bacterial culture. Such spatially homogeneous model was analyzed by Farein and Velarde[5] who found time-periodic solutions to explain the observed oscillations. Compared to the ODE system, the reaction-diffusion model (1.1) is more complicated and interesting, though they share some common conclusions. A general reaction-diffusion system, modeling a chemical reaction, has the form

$$
\frac{\partial \mathbf{u}}{\partial t}=D \Delta \mathbf{u}+F(\mathbf{u}, \mathbf{p})
$$

where $\mathbf{u}$ is a vector representing chemical concentrations, $\mathbf{p}$ is a vector of kinetic parameters, $D$ is a matrix of diffusion coefficients. Boundary conditions are usually taken as zero flux, that is, the domain boundary is assumed impermeable 
to the chemicals. In his seminal paper, Turing[12] proved, theoretically, that a system of type (1.2) could spontaneously evolve to spatially heterogeneous patterns from an initially uniform state in response to infinitesimal perturbations. Remarkably, he showed that diffusion could drive a chemical system to instability, leading to spatial patterns. Recently, the existence of Turing patterns in (1.1) has been investigated in [9, 7], exhibiting the critical role of the system parameters in leading to the formation of such structures. Model (1.1) has a quite similar mathematical form to the Lengyel-Epstein system

$$
\left\{\begin{array}{l}
\frac{\partial u}{\partial t}=\Delta u+a-u-\frac{4 u v}{1+u^{2}} \quad x \in \Omega, \quad t>0 \\
\frac{\partial v}{\partial t}=(\sigma c) \Delta v+(\sigma b)\left(u-\frac{u v}{1+u^{2}}\right)
\end{array}\right.
$$

describing the chlorite-iodide-malonic acid (CIMA) reaction[8]. The importance of system (1.3) is due to the fact that the first experimental evidence of the Turing pattern was observed on the CIMA reaction in an open unstirred gel reactor, almost 40 years after the publication of Turing's paper. Lengyel and Epstein[6] compared the Degn-Harrison model with the CIMA reaction model and found that the former can produce a richer dynamics. This paper devotes to the study of the asymptotic behavior of the dynamics generated by system (1.1). Our main aim is introducing suitable conditions on parameters of (1.1) ensuring the global attractivity of the unique constant steady-state solution. Obviously, the presence of global attractors excludes the formation of spatio-temporal patterns. The individuation of invariant rectangles is an important tool of our arguments. Indeed, the fact that such rectangles are also contracting, permits to replace, in some sense, the PDE system (1.1) by the corresponding ODE system with $d_{1}=d_{2}=0$, canceling, for $t$ sufficiently large, the $x$-dependence as shown in Theorem 5.1. The technique of contracting rectangles is illustrated in $[10,11]$ to obtain relevant global existence theorems. Another relevant tool is the method of Lyapunov, we employed in Theorem 5.3. Also in [3], the authors combine the technique of invariant rectangles with Lyapunov functionals to prove the convergence to a spatial homogeneous equilibrium solution, in a diffusion Lotka-Volterra system. The dynamics of the kinetic system

$$
\left\{\begin{array}{l}
u^{\prime}=a-u-\frac{u v}{1+k u^{2}} \\
v^{\prime}=b-\frac{u v}{1+k u^{2}}
\end{array}\right.
$$

is studied in Section 3, through the analysis of its isoclines in the phase-space, the Dulac criterion and the Poincaré-Bendixson theorem. The global stability of its unique equilibrium is investigated in Theorem 3.2. 


\section{Preliminaries}

We consider the following reaction-diffusion system based on the Degn-Harrison model

$$
\left\{\begin{array}{l}
\frac{\partial u}{\partial t}=d_{1} \Delta u+a-u-\phi_{k}(u) v \quad x \in \Omega, \quad t>0 \\
\frac{\partial v}{\partial t}=d_{2} \Delta v+b-\phi_{k}(u) v
\end{array}\right.
$$

with Neumann boundary condition

$$
\frac{\partial u}{\partial n}=\frac{\partial v}{\partial n}=0 \quad \text { on } \quad \partial \Omega
$$

and nonnegative initial value

$$
u(0, x)=u_{0}(x), \quad v(0, x)=v_{0}(x), \quad x \in \Omega
$$

where $u_{0}(x), v_{0}(x) \in C(\bar{\Omega}) \cap C^{2}(\Omega)$. In (2.1) the spatial domain $\Omega \subset \mathbf{R}^{n}$ is open, bounded and has regular boundary $\partial \Omega$, and

$$
\phi_{k}(u)=\frac{u}{1+k u^{2}}, \quad k>0 .
$$

The diffusion coefficients $d_{1}, d_{2}$ are positive, $d_{1} \neq d_{2}$, parameters $a, b$ are positive and $a>b$.

It is well known that $(2.1),(2.2),(2.3)$ possesses a unique classical solution $(u, v)$ with $u, v \in C^{1,2}\left(\mathbf{R}_{+}^{*} \times \Omega\right) \cap C\left(\mathbf{R}_{+} \times \bar{\Omega}\right)$. Such solution is positive, namely $u(t, x), v(t, x)>0$ for $t>0$ and $x \in \bar{\Omega}$. Henceforth we assume that the solutions of (2.1) are subject to (2.2) and (2.3).

It easy to see that (2.1) has a unique constant steady state

$$
\left(u^{*}, v^{*}\right), \quad u^{*}=a-b, \quad v^{*}=\frac{b}{\phi_{k}\left(u^{*}\right)}
$$

and we are interested in its global stability. For convenience of the reader, next we describe some results from $[7,9]$.

Theorem 2.1 [9] If $a^{2} k \leq 1$, then $\left(u^{*}, v^{*}\right)$ is a global attractor of (2.1) in the sense that, for any $(u(t, x), v(t, x))$ solution to (2.1)

$$
\lim _{t \rightarrow+\infty} u(t, x)=u^{*}, \quad \lim _{t \rightarrow+\infty} v(t, x)=v^{*}
$$

uniformly with respect to $x \in \bar{\Omega}$.

Taking into account previous theorem, afterwards we suppose

$$
a>\frac{1}{\sqrt{k}} .
$$

The below result regards the eventual (non constant) steady-state solutions of (2.1), that is the solutions of the elliptic boundary value problem

$$
\left\{\begin{array}{l}
-d_{1} \Delta u=a-u-\phi_{k}(u) v, \quad x \in \Omega \\
-d_{2} \Delta v=b-\phi_{k}(u) v \\
\frac{\partial u}{\partial n}=\frac{\partial v}{\partial n}=0 \quad \text { on } \quad \partial \Omega
\end{array}\right.
$$


Theorem 2.2 Let $(u(x), v(x))$ be any positive solution of (2.4) and assume that

$$
k a^{2}<\left(\frac{a}{b}-1\right) .
$$

Then the following estimates

$$
a\left(1-\frac{b}{\phi_{k}(a)}\right)<u(x)<a, \quad \frac{b}{a}<v(x)<\frac{b}{\phi_{k}(a)-b}, \quad x \in \bar{\Omega}
$$

hold.

The size of $\Omega$ and the diffusion coefficients play a central role in obtaining the nonexistence of steady-steady solutions. Roughly speaking, it follows from the investigations in $[9,7]$ that, under assumption $(2.5)$, if $d_{1}, d_{2}$ or the principal eigenvalue $\lambda_{1}$ are sufficiently large, then system (2.1) has no (non constant) steady-state solutions.

\section{The ODE system}

The model initially introduced by Degn and Harrison can be written as

$$
\left\{\begin{array}{l}
u^{\prime}=f(u, v) \\
v^{\prime}=g(u, v)
\end{array}\right.
$$

where $u(t), v(t)$ represent the concentration of oxygen and nutrient, respectively, and

$$
f(u, v)=a-u-\phi_{k}(u) v, \quad g(u, v)=b-\phi_{k}(u) v .
$$

It is obvious that non-negative solutions of model (3.1) are of real interest so that we take initial condition

$$
u(0)=u_{0}>0, \quad v(0)=v_{0}>0 .
$$

To study the local stability of $\left(u^{*}, v^{*}\right)$, we consider the Jacobian matrix $J(u, v)$ of (3.1). We find

$$
J\left(u^{*}, v^{*}\right)=\left(\begin{array}{cc}
-a_{11} & -a_{12} \\
-a_{11}+1 & -a_{12}
\end{array}\right)
$$

where

$$
a_{11}=1+\frac{b}{u^{*}} \frac{1-k\left(u^{*}\right)^{2}}{1+k\left(u^{*}\right)^{2}}, \quad a_{12}=\phi_{k}\left(u^{*}\right)>0 .
$$

Since det $J\left(u^{*}, v^{*}\right)=a_{12}$, one gets that $\left(u^{*}, v^{*}\right)$ is locally asymptotically stable for $(3.1)$ if

$$
\operatorname{trace} J\left(u^{*}, v^{*}\right)=-a_{11}-a_{12}=-\frac{a+(a-b)^{2}(1+k(a-2 b))}{(a-b)\left(1+k(a-b)^{2}\right)}<0 .
$$

Further informations about the dynamics of system (3.1) can be derived from the detailed analysis of its isoclines. The $v$-isocline

$$
\gamma_{v}: \quad v=\frac{b}{\phi_{k}(u)}
$$


has a minimum point at $\left(\frac{1}{\sqrt{k}}, 2 b \sqrt{k}\right)$ and goes to infinity for $u \rightarrow 0^{+}$. The $u$-isocline

$$
\gamma_{u}: \quad v=f_{a, k}(u), \quad f_{a, k}(u)=\frac{a-u}{\phi_{k}(u)}
$$

intersects the $u$-axes at $u=a$. It is strictly decreasing if $a^{2} k \leq 27$. If $a^{2} k>27$ $\gamma_{u}$ is strictly decreasing in $\left.] 0, \frac{1}{\sqrt{k}}\right]$ and $\left[\frac{a}{2}, a\right]$. In interval $] \frac{1}{\sqrt{k}}, \frac{a}{2}$ [ it has a local minimum $u_{1}$, a local maximum $u_{2}$ and a saddle point at $\sqrt[3]{\frac{a}{k}}$ with $u_{1}<\sqrt[3]{\frac{a}{k}}<$ $u_{2}$. The two isoclines intersect only at $\left(u^{*}, v^{*}\right)$ and $f_{a, k}(u)>\frac{b}{\phi_{k}(u)}$ for $0<u<$ $u^{*}$.

System (3.1) has an invariant rectangle in the phase plane, attracting all positive solutions, as shown in the following theorem. Put

$$
\mathcal{R}=[\bar{u}, a] \times[2 b \sqrt{k}, \bar{v}], \quad \bar{u}=\frac{b u^{*}}{a\left(1+k a^{2}\right)}, \quad \bar{v}=f_{a, k}(\bar{u}) .
$$

Theorem 3.1 Let $(u(t), v(t))$ be any solution of (3.1). Then there is a constant $T>0$, which may depend on $u_{0}$ and $v_{0}$, such that

$$
(u(t), v(t)) \in \mathcal{R} \quad \text { for all } t>T .
$$

Proof. As first step, we prove that the rectangle $\mathcal{R}$, defined in (3.5), is an invariant region. Since $b<a$ we are allowed to write $b=t a, 0<t<1$, so that

$$
\left.b u^{*}=t(1-t) a^{2} \leq \frac{a^{2}}{4}, \quad t \in\right] 0,1[.
$$

Consequently

$$
\bar{u} \leq \frac{a}{4\left(1+k a^{2}\right)}=\frac{\phi_{k}(a)}{4} \leq \frac{1}{8 \sqrt{k}}<\frac{1}{\sqrt{k}}
$$

because $\phi_{k}(u) \leq \frac{1}{2 \sqrt{k}}$. Observe that $f_{a, k}(u)$ and $\frac{b}{\phi_{k}(u)}$ are strictly decreasing in ] $0, \bar{u}]$ (for each $\mathrm{k}$ ).

As second step let us verify that

$$
\frac{b}{\phi_{k}(a)}<f_{a, k}(\bar{u})
$$

Indeed

$$
f_{a, k}(\bar{u})=\left(\frac{a}{\bar{u}}-1\right)\left(1+k \bar{u}^{2}\right)>\left(4\left(1+k a^{2}\right)-1\right)\left(1+k \bar{u}^{2}\right)>4 k a^{2}+3
$$

and

$$
\frac{b}{\phi_{k}(a)}=\frac{b}{a}\left(1+k a^{2}\right)<1+k a^{2} .
$$

Hence (3.7) easily follows. Previous estimates prove, in particular, that $\left(u^{*}, v^{*}\right)$ lies in the interior of $\mathcal{R}$. At this point, we can state that, on the boundary of $\mathcal{R}$, the vector field $(f(u, v), g(u, v))$, defined by $(3.2)$, does not point outwards. Indeed

$$
f(\bar{u}, v)>0 \quad \text { and } \quad f(a, v)<0 \quad \text { for } \quad 2 b \sqrt{k}<v<\bar{v}
$$




$$
g(u, 2 b \sqrt{k})>0 \quad \text { and } \quad g(u, \bar{v})<0 \quad \text { for } \quad \bar{u}<u<a .
$$

Therefore rectangle $\mathcal{R}$ is an invariant region.

Let $(u(t), v(t))$ be a positive solution to (3.1). It is obvious that

$$
u^{\prime}(t)<a-u(t) .
$$

Since all positive solutions of the ODE

$$
x^{\prime}(t)=a-x(t)
$$

tends to $a$ as $t \rightarrow+\infty$, by the comparison theorem,

$$
u(t) \leq a \text { for } t \text { sufficiently large } .
$$

Analogously, since $\phi_{k}(u) \leq \frac{1}{2 \sqrt{k}}$,

$$
v^{\prime}(t) \geq b-\frac{v(t)}{2 \sqrt{k}} .
$$

Taking into account that all solutions of the differential equation

$$
y^{\prime}(t)=b-\frac{y(t)}{2 \sqrt{k}}
$$

approach $2 b \sqrt{k}$ as $t \rightarrow+\infty$, we get

$$
v(t) \geq 2 b \sqrt{k} \text { for } t \text { sufficiently large . }
$$

Denote by $\mathcal{Q}$ the region $] 0, a] \times[2 b \sqrt{k},+\infty[$. Since

$$
\frac{b}{\phi_{k}(u)}<f_{a, k}(u) \text { if } u<u^{*},
$$

we can divide the region $\mathcal{Q} \backslash \mathcal{R}$ in three parts:

$$
\begin{aligned}
& \text { I) } \quad(u, v) \in \mathcal{Q} \backslash \mathcal{R}, \quad 0<u<\bar{u}, v \leq \frac{b}{\phi_{k}(u)} ; \\
& \text { II) } \quad(u, v) \in \mathcal{Q} \backslash \mathcal{R}, \quad v \geq f_{a, k}(u) ; \\
& \text { III) } \quad(u, v) \in \mathcal{Q} \backslash \mathcal{R}, \quad 0<u<\bar{u}, \frac{b}{\phi_{k}(u)}<v<f_{a, k}(u) .
\end{aligned}
$$

The trajectories starting in region $I$ ) have both components strictly increasing then they go into $\mathcal{R}$, at some $t$, otherwise they get into region $I I I$ ) after intersecting isocline $\gamma_{v}$. Similarly, the trajectories starting in region $I I$ ) have both components strictly decreasing then, after intersecting the line $v=f_{a, k}(\bar{u})$, they go into $\mathcal{R}$ or they intersect isocline $\gamma_{u}$ and enter into region $\left.I I I\right)$. Considering the direction of the vector field on isoclines $\gamma_{u}$ and $\gamma_{v}$, we deduce that the trajectories starting in region $I I I$ ) cannot leave this region till they go inside $\mathcal{R}$, for some $t$. The proof is complete.

We are ready to state and prove the main result in this section. 
Theorem 3.2 Let us suppose that

$$
3 \sqrt[3]{a k^{2}}-a k+1>0 .
$$

Then the equilibrium $\left(u^{*}, v^{*}\right)$ is globally asymptotically stable in the first quadrant as solution to (3.1).

Proof. The first step of our proof eliminates the existence of periodic solutions in $\mathcal{R}$. Rewrite system (2.1) in the form

$$
\left\{\begin{array}{l}
u^{\prime}=\phi_{k}(u)\left(f_{a, k}(u)-v\right), \quad t>0 \\
v^{\prime}=\phi_{k}(u)\left(\frac{b}{\phi_{k}(u)}-v\right) .
\end{array}\right.
$$

Obviously

$$
\frac{\partial}{\partial u}\left(f_{a, k}(u)-v\right)+\frac{\partial}{\partial v}\left(\frac{b}{\phi_{k}(u)}-v\right)=f_{a, k}^{\prime}(u)-1 .
$$

It easy to see that

$$
f_{a, k}^{\prime}(u)=-\frac{a}{u^{2}}+k a-2 k u
$$

is strictly negative for $a^{2} k \leq 27$. If $a^{2} k>27$, it is enough to prove

$$
f_{a, k}^{\prime}\left(\sqrt[3]{\frac{a}{k}}\right)-1<0
$$

to provide inequality $f_{a, k}^{\prime}(u)-1<0$ because $f_{a, k}^{\prime}(u)$ attains its maximin value at $u=\sqrt[3]{\frac{a}{k}}$. The equality

$$
f_{a, k}^{\prime}\left(\sqrt[3]{\frac{a}{k}}\right)-1=-3 \sqrt[3]{a k^{2}}+k a-1
$$

and assumption (3.8) exclude the presence of closed orbits, lying entirely in $\mathcal{R}$, by the Dulac criterion. To complete the proof it suffices to show that, under condition $(3.8),\left(u^{*}, v^{*}\right)$ is asymptotically stable. By (3.4), it is enough to demonstrate that inequality

$$
a+(a-b)^{2}(1+k(a-2 b))>0
$$

holds. We may assume $a<2 b$ because the validity of (3.10) is evident for $a \geq 2 b$. Introduce the function

$$
g(x)=\frac{a}{(a-x)^{2}}-(2 x-a) k+1 .
$$

We are going to prove that, under assumption (3.8),

$$
g(x)>0, \quad \frac{a}{2}<x<a .
$$


It easy to check that

$$
g\left(\frac{a}{2}\right)=\frac{4}{a}+1>0, \quad \lim _{x \rightarrow a^{-}} g(x)=+\infty
$$

and

$$
g^{\prime}(x)=\frac{2 a}{(a-x)^{3}}-2 k
$$

is strictly positive if and only if $x>a-\sqrt[3]{\frac{a}{k}}$. When $a^{2} k \leq 8$, it turns out $a-\sqrt[3]{\frac{a}{k}} \leq \frac{a}{2}$ so that $g(x)$ is strictly increasing in interval $] \frac{a}{2}, a[$. As a consequence

$$
g(x)>g\left(\frac{a}{2}\right)>0, \quad \frac{a}{2}<x<a .
$$

Let us consider the case $a^{2} k>8$. The point $a-\sqrt[3]{\frac{a}{k}}$ is a minimum for $g(x)$,

$$
g\left(a-\sqrt[3]{\frac{a}{k}}\right)=3 \sqrt[3]{a k^{2}}-a k+1
$$

therefore, using (3.8),

$$
g(x) \geq g\left(a-\sqrt[3]{\frac{a}{k}}\right)>0, \quad \frac{a}{2}<x<a .
$$

and (3.12) is proved. It implies that $a+(a-b)^{2}(1-(2 b-a) k)>0$ for each $b \in] \frac{a}{2}, a[$. Using the absence of periodic solutions and the Poincaré-Bendixson theorem, we complete the proof.

\section{Contracting rectangles as attracting regions}

Let

$$
0=\lambda_{0}<\lambda_{1}<\lambda_{2}<\ldots<\lambda_{i}<\ldots
$$

be the sequence of eigenvalues for the elliptic operator $(-\Delta)$, subject to the Neumann boundary conditions. Consider the linearized operator evaluated at $\left(u^{*}, v^{*}\right)$

$$
\left(\begin{array}{cc}
d_{1} \Delta-a_{11} & -a_{12} \\
1-a_{12} & d_{2} \Delta-a_{12}
\end{array}\right) .
$$

By Theorem 1[1], $\left(u^{*}, v^{*}\right)$ is asymptotically stable for (2.1) if, for each integer $i$, the eigenvalues of matrix

$$
M_{i}=\left(\begin{array}{cc}
-a_{11}-d_{1} \lambda_{i} & -a_{12} \\
1-a_{12} & -a_{12}-d_{2} \lambda_{i}
\end{array}\right)
$$

have negative real parts. Since

$$
\operatorname{det} M_{i}=d_{2} \lambda_{i}\left(d_{1} \lambda_{i}+a_{11}\right)+a_{12}\left(d_{1} \lambda_{i}+1\right)>0
$$


and

$$
\text { trace } M_{i}=-\left(d_{1}+d_{2}\right) \lambda_{i}-a_{11}-a_{12},
$$

we deduce that $\left(u^{*}, v^{*}\right)$ is certainly asymptotically stable when (3.10) holds. Our results on the global stability of $\left(u^{*}, v^{*}\right)$ depend on the existence of suitable invariant regions.

Definition 4.1 A closed subset $\Sigma \subset \mathbf{R}^{2}$ is called a (positively) invariant region for (2.1) if, any solution $(u(t, x), v(t, x))$ such that $u(0, x), v(0, x) \in \Sigma$ for all $x \in \Omega$, satisfies $(u(t, x), v(t, x)) \in \Sigma$ for all $t>0$ and $x \in \Omega$.

Definition $4.2 A$ rectangle $\Sigma=\left[u_{1}, u_{2}\right] \times\left[v_{1}, v_{2}\right]$ is contracting for the vector field $(p(u, v), q(u, v))$ if $(p, q)$ points into $\Sigma$ on the boundary $\partial \Sigma$, that is

$$
\begin{array}{lllll}
p\left(u_{1}, v\right)>0 & \text { and } & p\left(u_{2}, v\right)<0 & \text { for } & v_{1}<v<v_{2} \\
q\left(u, v_{1}\right)>0 & \text { and } & q\left(u, v_{2}\right)<0 & \text { for } & u_{1}<u<u_{2} .
\end{array}
$$

It can be shown (see[11]) that for a set to be invariant for PDE system (2.1) it must be a rectangle which is invariant for ODE system (2.5). Hence a contracting rectangle for the vector field $(f(u, v), g(u, v))$, defined by $(2.4)$, is an invariant region for system (2.1).

The next theorem shows the attractivity of rectangle $\mathcal{R}$ also for the solutions of reaction-diffusion system (2.1).

Theorem 4.1 Let $\mathcal{R}$ be the rectangle defined in (3.5). Then, for any positive solution $(u(t, x), v(t, x))$ of (2.1), there exists $T>0$ such that

$$
(u(t, x), v(t, x)) \in \stackrel{\circ}{\mathcal{R}}
$$

for $t>T$ and $x \in \Omega$.

Proof. As showed in Theorem 3.1, $\left(u^{*}, v^{*}\right)$ lies in the interior of $\mathcal{R}$ and

$$
\begin{aligned}
& f(\bar{u}, v)>0 \quad \text { and } \quad f(a, v)<0 \quad \text { for } \quad 2 b \sqrt{b}<v<\bar{v}, \\
& g(u, 2 b \sqrt{b})>0 \text { and } g(u, \bar{v})<0 \text { for } \bar{u}<u<a .
\end{aligned}
$$

Hence $\mathcal{R}$ is a contracting rectangle for the vector field $(f, g)$ by Definition 4.2. Since one can construct arbitrarily large contracting rectangles of this form, by Theorem 14.19[11], all solutions of (2.1) must enter into the invariant region $\mathcal{R}$ in finite time.

As a consequence of the previous theorem, $\mathcal{R}$ has to contain all eventual steady state solutions.

Corollary 4.1 Let $(u(x), v(x))$ be any positive solution of (2.4), then

$$
\bar{u}<u(x)<a, \quad 2 b \sqrt{k}<v(x)<\bar{v}, \quad x \in \Omega .
$$


In this way Theorem 2.2 is improved because we do not require the restrictive assumption (2.5).

Observe that, taking into account Theorem 2.1, condition (2.5) makes sense only if

$$
\frac{a}{b}-1>1
$$

that is $a>2 b$. For such values of $a$ and $b$, the solution $\left(u^{*}, v^{*}\right)$ is asymptotically stable because (3.10) holds.

\section{Global asymptotic stability}

In this section we investigate the global dynamics of the Degn-Harrison system (2.1).

In the next theorem we show that the solutions of (2.1) have the same asymptotic behavior of those to system (3.1) when they become spatially homogeneous for $t \rightarrow+\infty$.

First introduce some notations.

The Jacobian matrix of $(f(u, v), g(u, v))$ is given by

$$
J(u, v)=\left(\begin{array}{cc}
-\left(1+\frac{1-k u^{2}}{\left(1+k u^{2}\right)^{2}} v\right) & -\phi_{k}(u) \\
-\frac{1-k u^{2}}{\left(1+k u^{2}\right)^{2}} v & -\phi_{k}(u)
\end{array}\right) .
$$

Denote by $J^{s}(u, v)$ its corresponding symmetric matrix, that is

$$
J^{s}(u, v)=\left(\begin{array}{cc}
-\left(1+\frac{1-k u^{2}}{\left(1+k u^{2}\right)^{2}} v\right) & -\frac{1}{2}\left(\phi_{k}(u)+\frac{1-k u^{2}}{\left(1+k u^{2}\right)^{2}} v\right) \\
-\frac{1}{2}\left(\phi_{k}(u)+\frac{1-k u^{2}}{\left(1+k u^{2}\right)^{2}} v\right) & -\phi_{k}(u)
\end{array}\right) .
$$

Let $\mu(u, v)$ be the largest (real) eigenvalue of $J^{s}(u, v)$, then

$$
<\left(\begin{array}{l}
u \\
v
\end{array}\right), J^{s}(u, v) \cdot\left(\begin{array}{l}
u \\
v
\end{array}\right)>\leq \mu(u, v)\left(u^{2}+v^{2}\right) \leq \gamma\left(u^{2}+v^{2}\right), \quad(u, v) \in \mathcal{R}
$$

where

$$
\gamma=\max _{(u, v) \in \mathcal{R}} \mu(u, v) .
$$

Theorem 5.1 Let us suppose that (3.8) holds and

$$
\lambda_{1} d>\gamma, \quad d=\min \left\{d_{1}, d_{2}\right\}
$$

where, as before, $\lambda_{1}$ is the principal eigenvalue of $(-\Delta)$ and $\gamma$ is defined by (5.2). Then, for any solution $(u(t, x), v(t, x))$ of (2.1), we get

$$
\lim _{t \rightarrow+\infty}\left\|u(t, \cdot)-u^{*}\right\|_{L^{2}(\Omega)}=0=\lim _{t \rightarrow+\infty}\left\|v(t, \cdot)-v^{*}\right\|_{L^{2}(\Omega)} .
$$


Proof. Theorem 4.1 ensures the existence of $T>0$ such that $(u(t, x), v(t, x))$ lies in $\stackrel{\circ}{\mathcal{R}}$ for $x \in \Omega$ and $t>T$.

It is convenient to write (2.1) in the vectorial form

$$
z_{t}=D \Delta z+F(z)
$$

where

$$
D=\left(\begin{array}{cc}
d_{1} & 0 \\
0 & d_{2}
\end{array}\right), \quad z=(u, v), \quad F(z)=\left(\begin{array}{c}
a-u-\phi_{k}(u) v \\
b-\phi_{k}(u) v
\end{array}\right) .
$$

For $t>T$, put

$$
\Phi(t)=\frac{1}{2}\|\nabla z(t, \cdot)\|_{L^{2}(\Omega)}^{2}=\frac{1}{2} \int_{\Omega}<\nabla z(t, x), \nabla z(t, x)>d x
$$

where the inner product $\langle\cdot, \cdot\rangle$ is defined by

$$
<\nabla z, \nabla w>=\sum_{i=1}^{2} \sum_{j=1}^{n} z_{x_{j}}^{i} w_{x_{j}}^{i}, \quad z=\left(z^{1}, z^{2}\right), w=\left(w^{1}, w^{2}\right) .
$$

Known arguments (see[2, 8]) yield

$$
\begin{gathered}
\frac{d \Phi}{d t}=\int_{\Omega}<\nabla z, \nabla z_{t}>d x=\int_{\Omega}<\nabla z, \nabla(D \Delta z)>d x+\int_{\Omega}<\nabla z, \nabla F(z)>d x \\
=-\int_{\Omega}<\Delta z, D \Delta z>d x+\int_{\Omega}<\nabla z, J(z) \cdot \nabla z>d x \\
\leq-d \int_{\Omega}|\Delta z|^{2} d x+\int_{\Omega}<\nabla z, J^{s}(z) \cdot \nabla z>d x
\end{gathered}
$$

In the light of Lemma A.1[2]

$$
\|\Delta z\|_{L^{2}(\Omega)}^{2} \geq \lambda_{1}\|\nabla z\|_{L^{2}(\Omega)}^{2},
$$

hence, by (5.1)

$$
\frac{d \Phi}{d t} \leq-d \lambda_{1} \int_{\Omega}|\nabla z|^{2} d x+\gamma \int_{\Omega}|\nabla z|^{2} d x=-2\left(d \lambda_{1}-\gamma\right) \Phi
$$

which implies the existence of a constant $c>0$ such that

$$
\|\nabla z\|_{L^{2}(\Omega)} \leq c e^{-\left(d \lambda_{1}-\gamma\right) t}, \quad t>T .
$$

Estimate (5.5) implies that all solutions of (2.1), with values in $\mathcal{R}$, converge to their spatial averages $\bar{z}(t)$ over $\Omega$, i.e.

$$
\bar{z}(t)=\frac{1}{|\Omega|} \int_{\Omega} z(t, x) d x .
$$


In fact the Poincaré inequality gives

$$
\|z(t, \cdot)-\bar{z}(t)\|_{L^{2}(\Omega)}^{2} \leq \frac{1}{\lambda_{1}}\|\nabla z(t, \cdot)\|_{L^{2}(\Omega)}^{2}
$$

thus, by (5.3) and (5.5), one deduces

$$
\lim _{t \rightarrow+\infty}\|z(t, \cdot)-\bar{z}(t)\|_{L^{2}(\Omega)}=0 .
$$

The last part of the proof shows that, under our assumptions, the asymptotic behavior of (2.1) is determined only by the corresponding ODE system (3.1). From Theorem 3.1[2] we obtain that $\bar{z}(t)=(\bar{u}(t), \bar{v}(t))$ satisfies the ODE system

$$
\left\{\begin{array}{l}
u^{\prime}=f(u, v)+q_{1}(t) \\
v^{\prime}=g(u, v)+q_{2}(t)
\end{array}\right.
$$

with initial conditions

$$
u(0)=\frac{1}{|\Omega|} \int_{\Omega} u_{0}(x) d x, \quad v(0)=\frac{1}{|\Omega|} \int_{\Omega} v_{0}(x) d x,
$$

where, for some $c>0$,

$$
\left|q_{i}(t)\right| \leq c e^{-\left(d \lambda_{1}-\gamma\right) t}, \quad t>T .
$$

Hence, by (5.3), the solutions of (5.6) have the same asymptotic behavior of those of system (3.1) (see [8]). Since (3.8) holds, we can apply Theorem 3.2, so that $\left(u^{*}, v^{*}\right)$ is a global attractor for system (3.1) in $\mathbf{R}_{+}^{2}$. We deduce

$$
\lim _{t \rightarrow+\infty}\left|\bar{u}(t)-u^{*}\right|=0=\lim _{t \rightarrow+\infty}\left|\bar{v}(t)-v^{*}\right|
$$

and (5.4) easily follows.

Remark 5.1 We wish to stress the fact, that if the matrix $J^{s}(u, v)$ is negative definite in $\mathcal{R}$, then the conclusion of Theorem 5.1 is valid, provided that only (3.8) holds.

When inequality (3.8) fails, we are able to provide the global asymptotic stability of $\left(u^{*}, v^{*}\right)$ in a smaller rectangle $\mathcal{T} \subset \mathcal{R}$ under suitable constrains only on parameters $a, b, k$ and no restrictions on the size of $\Omega$ (due to $\lambda_{1}$ ) and the diffusion coefficients $d_{1}, d_{2}$.

Theorem 5.2 Suppose that $3 \sqrt[3]{a k^{2}}-a k+1 \leq 0$ and

$$
b \leq \frac{a}{4}\left(\frac{4+a^{2} k}{1+a^{2} k}\right) .
$$

Then the rectangle

$$
\mathcal{T}=\left[\frac{a}{2}, a\right] \times\left[2 b \sqrt{k}, f_{a, k}\left(\frac{a}{2}\right)\right],
$$

is an invariant region for system (2.1). 
Proof. The first assumption implies that $a^{2} k>27$. It turns out that inequality $\frac{4+a^{2} k}{1+a^{2} k}<\frac{4}{3}$ holds. Using (5.7) one deduces

$$
b<\frac{a}{3} \quad \text { and } \quad u^{*}=a-b>\frac{2}{3} a .
$$

Consequently, under our assumptions, $\left(u^{*}, v^{*}\right) \in \mathcal{T}$, isocline $\gamma_{u}$ is strictly decreasing, isocline $\gamma_{v}$ is strictly increasing in rectangle $\mathcal{T}$. Moreover

$$
\frac{b}{\phi_{k}(a)} \leq f_{a, k}\left(\frac{a}{2}\right) \quad \text { and } \quad \mathcal{T} \subset \mathcal{R} \text {. }
$$

It is not difficult to verify that the vector field $(f, g)$, defined by $(3.2)$, does not point outwards on the boundary of $\mathcal{T}$, that is, by Definition $3.2, \mathcal{T}$ is contracting. Hence $\mathcal{T}$ is an invariant region for system (2.1).

Theorem 5.2 leads to the following one.

Theorem 5.3 Suppose that the assumptions of Theorem 5.2 hold. Let $(u(t, x), v(t, x))$ be any solution of (2.1) having its initial value in rectangle $\mathcal{T}$, defined by (5.8), for all $x \in \Omega$. Then

$$
\lim _{t \rightarrow+\infty} u(t, x)=u^{*}, \quad \lim _{t \rightarrow+\infty} v(t, x)=v^{*}
$$

uniformly in $x \in \Omega$.

Proof. Take $(u(t, x), v(t, x))$, solution of (2.1) with initial value $\left(u_{0}(x), v_{0}(x)\right) \in$ $\mathcal{T}$, for all $x \in \Omega$. By Theorem 5.2, it turns out that

$$
(u(t, x), v(t, x)) \in \mathcal{T}, \quad t>0, x \in \Omega .
$$

It is convenient to write system (2.1) in the form

$$
\left\{\begin{array}{l}
\frac{\partial u}{\partial t}=d_{1} \Delta u+\phi_{k}(u)\left[\left(f_{a, k}(u)-f_{a, k}\left(u^{*}\right)\right)-\left(v-v^{*}\right)\right] \\
\frac{\partial v}{\partial t}=d_{2} \Delta v+\phi_{k}(u)\left[\left(\frac{b}{\phi_{k}(u)}-\frac{b}{\phi_{k}\left(u^{*}\right)}\right)-\left(v-v^{*}\right)\right] .
\end{array}\right.
$$

Putting

$$
E(u, v)=\int_{u^{*}}^{u}\left(\frac{b}{\phi_{k}(s)}-\frac{b}{\phi_{k}\left(u^{*}\right)}\right) d s+\frac{\left(v-v^{*}\right)^{2}}{2},
$$

consider the Lyapunov function

$$
V(t)=\int_{\Omega} E(u(t, x), v(t, x)) d x .
$$

The time derivative of $V(t)$ along the solutions of system(2.1) yields

$$
V^{\prime}(t)=\int_{\Omega}\left[\left(\frac{b}{\phi_{k}(u)}-\frac{b}{\phi_{k}\left(u^{*}\right)}\right) \frac{\partial u}{\partial t}+\left(v-v^{*}\right) \frac{\partial v}{\partial t}\right] d x
$$




$$
\begin{gathered}
=\int_{\Omega} \phi_{k}(u)\left[\left(\frac{b}{\phi_{k}(u)}-\frac{b}{\phi_{h}\left(u^{*}\right)}\right)\left(f_{a, k}(u)-f_{a, k}\left(u^{*}\right)\right)-\left(v-v^{*}\right)^{2}\right] d x \\
+d_{1} \int_{\Omega}\left(\frac{b}{\phi_{k}(u)}-\frac{b}{\phi_{h}\left(u^{*}\right)}\right) \Delta u d x+d_{2} \int_{\Omega}\left(v-v^{*}\right) \Delta v d x .
\end{gathered}
$$

Taking into account the boundary conditions for $(u, v)$, we find

$$
\int_{\Omega}\left(v-v^{*}\right) \Delta v d x=-\int_{\Omega}|\nabla v|^{2} d x<0
$$

and analogously

$$
\int_{\Omega}\left(\frac{b}{\phi_{k}(u)}-\frac{b}{\phi_{h}\left(u^{*}\right)}\right) \Delta u d x=-\int_{\Omega}\left(\frac{b}{\phi_{k}(u)}\right)^{\prime}|\nabla u|^{2} d x .
$$

Also this second addendum of (5.10) is strictly negative because $\frac{b}{\phi_{k}(u)}$ is strictly increasing in $\left[\frac{a}{2}, a\right]$. In addition, since $f_{a, k}(u)$ is strictly decreasing in $\left[\frac{a}{2}, a\right]$, we get

$$
\begin{gathered}
u<u^{*} \Rightarrow\left(\frac{b}{\phi_{k}(u)}-\frac{b}{\phi_{k}\left(u^{*}\right)}\right)<0 \quad \text { and } \quad\left(f_{a, k}(u)-f_{a, k}\left(u^{*}\right)\right)>0 \\
u>u^{*} \Rightarrow\left(\frac{b}{\phi_{k}(u)}-\frac{b}{\phi_{k}\left(u^{*}\right)}\right)>0 \quad \text { and } \quad\left(f_{a, k}(u)-f_{a, k}\left(u^{*}\right)\right)<0 .
\end{gathered}
$$

Thus, going back to (5.10), we obtain that $V^{\prime}(t)$ is strictly negative for $(u, v) \neq$ $\left(u^{*}, v^{*}\right)$. It is easy to verify that $\left\{\left(u^{*}, v^{*}\right)\right\}$ is the largest invariant set of

$$
\left\{(u(t, x), v(t, x)) \in \mathcal{T} \mid V^{\prime}(t)=0\right\}
$$

hence known arguments (see[13]) lead to

$$
\lim _{t \rightarrow+\infty}\left|u(t, x)-u^{*}\right|=0=\lim _{t \rightarrow+\infty}\left|v(t, x)-v^{*}\right|
$$

uniformly for $x \in \Omega$.

\section{References}

[1] R.Casten, C.J.Holland, Stability properties of solutions to systems of reaction-diffusion equations, SIAM J.Appl.Math. 35 (1978) 353-364.

[2] E.Conway, D.Hoff, J.Smoller, Large time behavior of solutions of systems of nonlinear reaction-diffusion equations, SIAM J.Appl.Math. 33 (1978) 1-16.

[3] P.De Mottoni, F.Rothe, Convergence to homogeneous equilibrium state for generalized Volterra-Lotka systems with diffusion, SIAM J.Appl.Math. 37 (1979) 648-663.

[4] H.Degn, D.E.F.Harrison, Theory of oscillations of respiratory rate in continuous culture of Klebsiella aerogenes, J.Theoret.Biol. 22 (1969) 238-248. 
[5] V.Farein, M.G.Velarde, Time-periodic oscillations in a model for the respiratory process of a bacterial culture, J.Math.Biol. 8 (1979) 147-157.

[6] I.Lengyel, I.R.Epstein, Diffusion-induced instability in chemically reaction systems: steady-state multiplicity, oscillation, and chaos, Chaos 1 (1991) 69-76.

[7] S.Li, J.Wu, Y.Dong, Turing patterns in a reaction-diffusion model with the Degn-Harrison scheme, J.Differential Equations 259 (2015) 1990-2029.

[8] B.Lisena, On the global dynamics of the Lengyel-Epstein system, Appl.Math.Comput. 249 (2014) 67-75.

[9] R.Peng, F.Q.Yi, X.Q.Zhao, Spatiotemporal patterns in a reaction-diffusion model with the Degn-Harrison reaction scheme, J.Differential Equations 254 (2013) 2465-2498.

[10] J.Rauch, J.Smoller, Qualitative theory of the FitzHugh-Nagumo equations, Advances in Mathematics 27 (1978) 12-44.

[11] J.Smoller, Shock Waves and Reaction-Diffusion Equations, SpringerVerlag, New-York 1983.

[12] A.M.Turing, The chemical basis of morphogenesis, Philos.Trans.R.Soc.Lond.Ser.B 237 (1952) 37-72.

[13] F.Yi, J.Wei, J.Shi, Global asymptotic behavior of the Lengyel-Epstein reaction diffusion system, Appl.Math.Lett. 22 (2009) 52-55. 